\title{
Suppression of drinking following rotational stimulation as an index of motion sickness in the rat
}

\author{
VAHRAM HAROUTUNIAN, DAVID C. RICCIO, and DONALD P. GANS \\ Kent State University, Kent, Ohio 44242
}

\begin{abstract}
A suppression-of-drinking measure was used as an index of the duration and magnitude of rotationelicited UCR. Thirsty rats suppressed drinking when they were rotated prior to being given access to water. The magnitude of suppression increased as a function of the duration of intermittent rotation. Periodically interrupted rotation sessions were found to suppress drinking for longer periods than continuous rotation sessions of comparable duration. Damage to the vestibular system substantially decreased the magnitude of suppression of drinking following rotation and prevented the acquisition of rotation-induced taste aversion. The failure of rotation-induced taste aversion to develop in rats sustaining vestibular damage was not attributable to an associative/sensory deficit as these rats acquired the aversion when lithium chloride poisoning was used as the UCS.
\end{abstract}

Evidence from several studies indicates that rats, despite their inability to vomit, are relatively sensitive to the effects of rotational stimulation. Thus, rats exposed to modest rotation speeds of $10-15 \mathrm{rpm}$ show clear suppression of locomotor activity (Eskin \& Riccio, 1966). Similarly, a reduction in foodreinforced leverpresses occurs when rotation is superimposed upon otherwise stable levels of responding generated by basic ratio or interval schedules (Riccio \& Thach, 1968). Rats subjected to rapid spinning following ingestion of a novel-flavored substance develop taste aversion to the flavor (Braun \& McIntosh, 1973; Green \& Rachlin, 1973), and, as in the case with more traumatic agents such as poisoning, the learning can occur despite an appreciable delay between the CS and UCS (Green \& Rachlin, 1976; Haroutunian \& Riccio, 1975). Taken together, these findings suggest that rotation serves as a stimulus which produces motion sickness or malaise in the rat.

One methodological issue raised by these findings concerns the extent to which the illness persists after termination of the physical stimulus. Eskin and Riccio (1966) reported data which appeared to indicate that recovery of activity following slow rotation treatment, while not immediate, was nevertheless quite rapid. It would seem to be of some interest to specify more precisely the duration of the elicited UCR. More generally, information on temporal aspects of sickness may provide an indirect index for assessing the magnitude of the UCR, especially in

This study was supported in part by NSF Grant GB41488 to David C. Riccio. The authors acknowledge the helpful assistance of Gay Phillips in data collection. Reprint requests should be sent to David C. Riccio, Department of Psychology, Kent State University, Kent, Ohio 44242. those cases where behavioral measurement is not feasible during ongoing treatment. With other parameters held constant, duration of illness should persist longer after more intense stimulation.

\section{EXPERIMENT 1}

In this experiment, we attempted to assess sickness by measuring the extent to which a prepotent class of responses was disrupted following rotational treatment: mildly thirsty rats, previously trained to approach a drinking spout, were tested for their latency to begin drinking following various rotational treatments. Presumably, the onset of drinking would represent a common threshold for all groups, i.e., the point at which illness has subsided sufficiently for thirst-motivated responding to be expressed. The major aims of the experiment were to determine whether suppression of drinking would occur following rotation exposure, and, if so, whether the extent of suppression would vary as a function of the magnitude (duration) of treatment.

In the standard exposure conditions here, as well as in our previous study (Haroutunian \& Riccio, 1975), rotation was periodically interrupted to provide a series of accelerations and decelerations. It was assumed that these abrupt changes in speed would provide more intense vestibular stimulation as well as reduce any sensory adaptation which might occur during exposure to constant angular velocities. An ancillary aim here was to check this assumption by comparing the amount of disruption produced by continuous and intermittent rotation conditions.

\footnotetext{
Method

Subjects. Four groups of eight adult male albino rats were used in this experiment. The rats were housed in individual cages and
} 
had continuous access to food. Access to water was gradually decreased to $1 \mathrm{~h}$ a day over a 7-day period.

Apparatus. A phonograph turntable rotating at approximately $70 \mathrm{rpm}$ served as the rotation device. Rotation periods were controlled by electromechanical programming equipment located in a room adjacent to the experiment room. A Universal timer (Dimco-Gray Co., Dayton, Ohio) was used to measure the latency to begin drinking.

Procedure. Following Day 7 of adaptation to water deprivation, each subject was transported in its home cage to the experimental room, where it remained undisturbed for $10 \mathrm{~min}$. A water bottle was then inserted into the home cage, and the latency to begin drinking was recorded. Ten minutes later, each subject was returned to the colony and the water bottle was removed after $50 \mathrm{~min}$. This procedure was repeated for 4 consecutive days until a stable pretreatment baseline of latency to begin drinking was established. A 1-day rest followed, with subjects given $1 \mathrm{~h}$ access to water in their home cages. On the next day, each rat was taken to the experimental room, where it was rotated at $70 \mathrm{rpm}$. Groups of eight rats received one of the following rotation treatments: 101 -min rotations (Group 10-I), 5 1-min rotations (Group 5-I), or 2 1-min rotations (Group 2-I). The interrotation interval was $2.5 \mathrm{sec}$ for these groups. Group $10-\mathrm{C}$ received 110 -min continuous rotation treatment. Immediately following rotation, a water bottle was inserted into each cage and the subject's latency to start drinking was measured. This treatment was repeated on three separate occasions with 1-day rest periods in between.

\section{Results and Discussion}

The median latencies to begin drinking on the last day of baseline measurement and the subsequent postrotation drinking sessions for all treatment conditions are shown in Figure 1. Because of the skewed nature of the data, a Kruskal-Wallis nonparametric analysis of variance was used. It indicated that the four groups did not differ in their latencies to begin drinking on the last pretreatment baseline day $(H<1.0, p>.05)$. In contrast, rotation, even in the least effective condition of continuous stimulation (Group 10-C), increased latencies to begin drinking above that established on the last baseline day (Mann-Whitney $U$ test, $U=7.5, p<.005$ ).

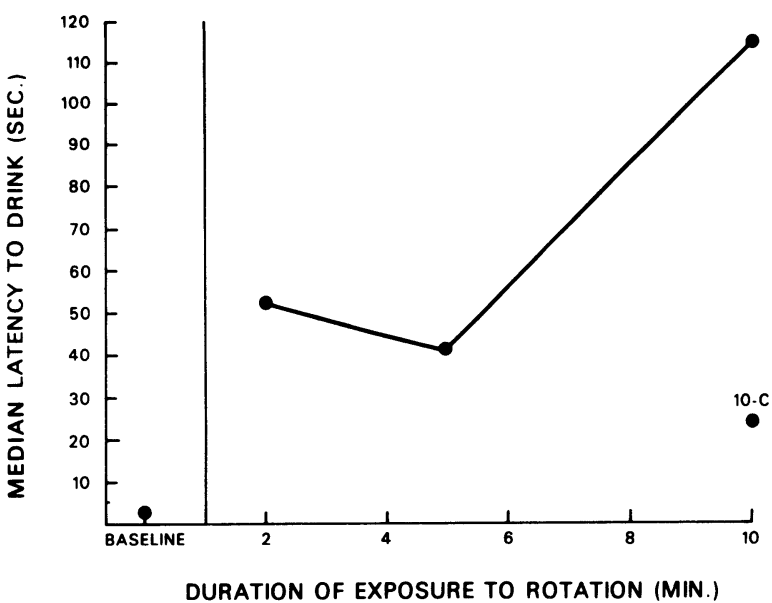

Figure 1. Median latencies to begin drinking during the baseline session and following 2-, 5-, and 10-min intermittent and 10-min continuous (10-C) rotation.
A Kruskal-Wallis analysis of postrotation latencies to begin drinking for all four groups, pooled over sessions, revealed a significant overall treatment effect $(\mathrm{H}=21.49, \mathrm{p}<.001)$. Mann-Whitney $\mathrm{U}$ tests showed that the long intermittent rotation treatment (Group 10-I) was more effective than shorter duration (2- and 5-min) intermittent rotation (ps $<.05$ ). Although subjects receiving 2- or 5-min-long intermittent rotation (Groups 2-I and 5-I, respectively) did not differ significantly $(U=32.0, p>.05)$, they began drinking after a longer delay than subjects which had received $10 \mathrm{~min}$ of continuous rotation (Group 10-C, ps <.05). These findings show that rapid accelerations and decelerations potentiate the effects of rotation, to the point of rendering $2 \mathrm{~min}$ of intermittent rotation more effective than $10 \mathrm{~min}$ of uninterrupted rotation treatment. It should be noted that, as the semicircular canals are acceleration rather than velocity detectors, even slight head movements made by the unrestrained rat during rotation at a constant speed generate accelerations in the vestibular system. It is these forces, as well as speed changes at onset and offset of rotation, which are effective in the continuous rotation condition. In any event, the present data demonstrate the utility of the postrotation disruption of drinking measure as a behavioral indicant of the UCR elicited by rotation.

When data from all four groups were pooled and analyzed for the effects of repeated rotation sessions, it was found that the duration of suppression decreased over sessions $\left(\chi \mathrm{r}^{2}=35.14, \mathrm{p}<.001\right)$. This effect of sessions appears attributable to a particularly potent disruption of drinking upon initial exposure to rotation: latencies to drink in Session 1 were significantly longer than in Session 2 or Session 3 (ps $<.05$ ), but the latter two sessions did not differ from each other $(Z=-1.32, p>.05)$.

\section{EXPERIMENT 2}

The preceding experiment demonstrated that latency to begin drinking following rotation can provide a measure of rotational severity. Presumably, the disruption of drinking is related to malaise or "motion sickness" elicited by the vestibular stimulation during rotation. Indirect support for this view comes from evidence that rats with partial vestibular damage show less suppression of activity during exposure to slow rotation speeds than do intact animals (Riccio, Igarashi, \& Eskin, 1967). As a more direct assessment of the contribution of the vestibular apparatus to these rotational effects, and as a check on the validity of the suppression of drinking measure, Experiment 2 compared rats with and without experimental destruction of the vestibular mechanisms. If the delay in drinking following rotation observed in Experiment 1 reflects vestibular mediation of illness, 
then damage to the vestibular area should eliminate, or reduce, postrotation disruption of drinking.

\section{Method}

Subjects and Surgery. Male albino rats $(250-320 \mathrm{~g})$ were anesthetized with $.3 \mathrm{mg} / \mathrm{kg}$ Equi-Thesin (Jensen-Salsbery Labs., Kansas City, Missouri) administered intraperitoneally. Once unconscious, each rat was placed in a head-holding device and was rotated approximately $90^{\circ}$ so that the external auditory meatus was visible with the aid of a speculum under a 30-power dissecting microscope. The meatus was washed with physiological saline and the pars flassida of the tympanic membrane was punctured and peeled away with a blunt needle. After repeating this procedure on the other ear, the procedure was terminated for the rats assigned to the sham control group $(\mathrm{N}=8)$. The external auditory meatus of each ear was packed with gelfoam, and these rats were returned to their home cages.

The operative procedure was continued for the rats assigned to the group whose vestibular system was to be destroyed $(\mathbf{N}=8)$. The malleus was nudged towards the posterior quadrant of the tympanic membrane and the incus was removed with a pair of fine forceps. The stapes crura was gently pushed away from the oval window to avoid puncturing the stapedial artery. The stapes crura was usually fractured at the stapes footplate. The footplate was carefully removed, exposing the oval window. The tip of the electrode was placed near the oval window and was gradually moved until the indentation of the oval window was felt under the tip. The electrode tip (.65 $\mathrm{mm}$ in diameter) was large enough to fit into the indentation of the oval window but too large to pass through it. The electrode was connected to a Birtcher hyfrecator, Model 732. The electrode tip was held firmly in place as the hyfrecator was activated for $10 \mathrm{sec}$, passing a mild monopolar high-frequency damped current (control dial set at 50). Following the passage of current, the electrode was removed and the external auditory meatus and the middle ear cavity were flooded with saline and aspirated several times to minimize any peripheral bleeding. The middle-ear cavity and external auditory meatus were packed with gelfoam, the subject was repositioned, and the same procedure was repeated on the second ear. The rat was then returned to its home cage. The entire procedure was always completed within $1 \mathrm{~h}$.

The subjects recovered from the anesthesia after approximately $4 \mathrm{~h}$. The rats with vestibular damage immediately displayed signs of vestibular dysfunction such as spinning when suspended by their tails, nystagmoid movements of the head, retropulsion, and turning in one direction then the next, when placed on the floor. All the subjects remained undisturbed in group cages for $\mathbf{3 0}$ days. By the end of this period they were able to locomote, eat, and drink even though they still displayed many of the abovementioned behavioral characteristics.

Thirty days following the operative procedure, the subjects in the vestibular damage group (Vest-Dam) and the sham-operated group (Sham-Op) were housed in single cages where they had continuous access to food. Water was gradually rationed over a 7-day period until it was available to each rat for only $15 \mathrm{~min}$ a day.

Apparatus. The rotation device described for Experiment 1 was also used in this experiment. Rotation periods were controlled by a Universal timer, which was also used to measure latency to start drinking. Rats were rotated inside regular wire-mesh cages which had white cardboard inserts covering all four walls. A small hole, $1 \mathrm{~cm}$ in diameter, was cut $2.5 \mathrm{~cm}$ above the floor on one wall of the cage to allow a water bottle spout to be inserted into the cage.

Procedure. After adaptation to water deprivation, a baseline measure of the latency to onset of drinking was taken for 4 days. Once a day, each rat was placed inside the test cage for $5 \mathrm{~min}$ and left undisturbed. At the end of this time, a water bottle was inserted into the cage and the latency to start drinking was recorded. The subject had $15 \mathrm{~min}$ of unrestricted access to water while in this distinctive cage. For the next 3 days, all animals received the same treatment, except that they were rotated for $5 \mathrm{~min}$ immediately prior to being presented with water. The rotation condition consisted of exposure to $70 \mathrm{rpm}$ for 1030 -sec intervals with an interrotation interval of $2.5 \mathrm{sec}$. Immediately following rotation, a water bottle was inserted into the cage and the latency to begin drinking recorded.

Following Day 3 of rotation, an accidental overheating of the animal colony occurred. Therefore, subjects were given ad-lib access to water for $24 \mathrm{~h}$ to minimize any stress which may have resulted. Two days of baseline drinking measures were taken before the rotation sessions were reinstituted. To insure that any changes occurring in drinking latency following rotation were not due to acquired aversive properties of the distinctive (white-lined) rotation chamber, a procedural change was also made at this point. Immediately following rotation in the distinctive chamber, the subjects were returned to their home cage, where the water bottle was made available. Latency to start drinking was measured as before. This procedure was repeated for 2 days.

\section{Results and Discussion}

As Figure 2 suggests, a significant difference in the latency to begin drinking developed between the Sham-Op and Vest-Dam groups following exposure to rotation $(F=20.14, p<.01)$. This difference in response latency persisted throughout the experiment and was not affected by the interruption caused by overheating or by the change in apparatus where testing occurred. The two groups differed significantly even on the last rotation session, where the separation between them was least $(t=2.14, p<.05)$. Equally important, the two groups differed in their latency to begin drinking only when they were rotated, as no differences in drinking latencies were apparent on the last day of baseline measurement taken prior to rotation $(\mathrm{t}=1.06$, $p>.05)$. Furthermore, the baseline taken following interruption of the experiment did not differ from the original baseline measure $(t=1.17, p>.05)$. These findings support our expectations that rats sustaining vestibular damage would be minimally affected by rotation when compared with intact animals.

A significant rotation sessions effect $(F=5.60$, $\mathrm{p}<.01)$, as well as a Treatment by Rotation inter-

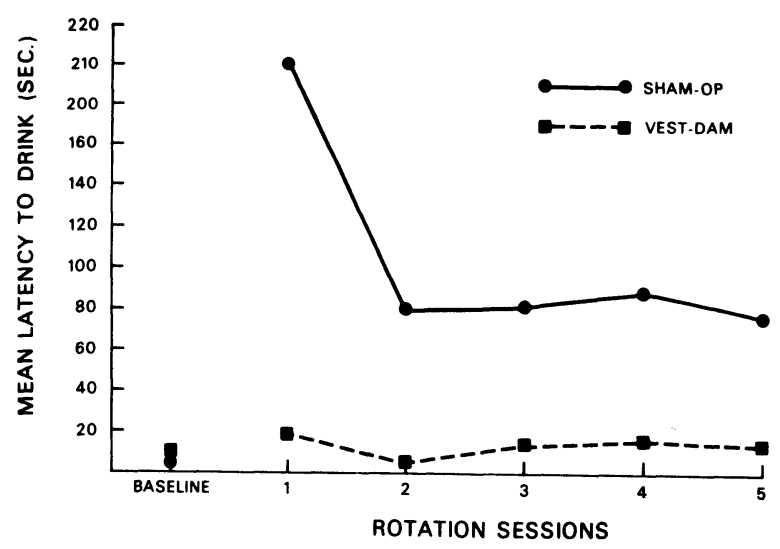

Figure 2. Mean latencies to begin drinking by Vest-Dam and Sham-Op subjects following $10 \mathrm{~min}$ of intermittent rotation. 
action $(F=36.51, p<.01)$, was also found. These differences are clearly evident in Figure 2, which shows that both the rotation sessions effect and the Treatment by Rotation Sessions interaction were a result of a drop in response to rotation by the Sham-Op group following Session 1. As in Experiment 1 , the decline in the intensity of response to rotation in the intact rats occurred following the first rotation session. This finding suggests that the initial response to rotation by these rats was potentiated by a novelty effect which diminished after the first experience with rotation.

\section{EXPERIMENT 3a}

Damage to the vestibular system appears to render rats, like other species (e.g., Johnson, Meek, \& Graybiel, 1962; Wang \& Chinn, 1956), relatively immune to motion sickness. Consistent with this interpretation is the finding that rotation failed to suppress drinking behavior of operated rats in Experiment 2 , and a previous observation that rotation is ineffective in producing taste aversion in vestibular damaged subjects (Haroutunian \& Riccio, 1975). As the latter data were reported only incidentally, it seemed appropriate to provide more direct information on this issue by utilizing the present subjects in a taste aversion paradigm. While employing the same two groups of animals makes efficient use of the subject sample, there is evidence that prior experience with the UCS may attenuate acquisition of taste aversion (Braveman, 1975; Cannon, Berman, Baker, \& Atkinson, 1975). In order to evaluate this possibility, an additional group of intact rats with no previous rotation experience was included in the design.

\section{Method}

Subjects. The two groups from the previous experiment were maintained on a deprivation schedule of access to water for $15 \mathrm{~min}$ a day during the 1-week interval between Experiments 1 and 2. Eight additional rats were used as a control group, and were gradually water deprived to $15 \mathrm{~min}$ access daily. Animals were housed in individual cages where food was freely available.

Apparatus. The turntable described for Experiments 1 and 2 was also used in this experiment. In addition, a number of large cages $(41.9 \times 21.10 \times 14.6 \mathrm{~cm})$ were used for testing choice preference for a $0.1 \%(1 \mathrm{~g} /$ liter $)$ saccharin solution.

Procedure. One week following the completion of Experiment 1, each subject in the vestibular damage, sham control, and no prerotation control (NPRC) groups received 4 days of two-bottle preference tests for saccharin vs. water. Each rat was placed inside a large cage for $15 \mathrm{~min}$ with a bottle of saccharin solution and one of water inserted at opposite ends of the cage. The bottles were weighed before and after consumption, and the percentage of saccharin of the total liquid consumed was recorded. This procedure was repeated for 4 days or until a saccharin preference criterion of $70 \%$ of total liquid consumed was reached.

Once this criterion was reached, the subjects received a 15-min single-bottle saccharin drinking session in their home cage. Immediately following saccharin consumption, the rats were placed on the rotation turntable while in their home cage and were rotated at $70 \mathrm{rpm}$ for 101 -min intervals with an interrotation interval of
$2.5 \mathrm{sec}$. On the next day, all subjects were tested for saccharin vs. water preference in the two-bottle situation described earlier. On the remaining 2 days of this 4-day cycle, the subjects received water only and were taken to the experimental room but not rotated. This 4-day treatment-test procedure was repeated five times.

\section{Results and Discussion}

Figure 3 presents the data on saccharin preference during baseline sessions and following rotation for the three groups. No marked changes occurred in the saccharin preference of the Vest-Dam group as a result of rotation, while control (NPRC) rats significantly reduced their intake of saccharin over the course of the saccharin-rotation pairings. An analysis of variance revealed a significant treatments effect $(F=6.79, p<.005)$, a significant rotation sessions effect $(F=5.60, p<.001)$, but a nonsignificant Rotation Sessions by Treatments interaction $(F=1.89, p>.05)$. Comparisons using NewmanKeuls tests showed that subjects with vestibular damage consumed significantly more saccharin than controls (NPRC) which had not previously been rotated (ps $<.05$ ) on all test sessions but the first. The saccharin preference of Vest-Dam rats did not depart significantly from their initial baseline levels on any of the test sessions, suggesting that vestibular damage eliminates the malaise ordinarily induced by rotation treatment. Moreover, a similar, but less robust, protection was afforded by prior rotational experience (Sham-Op). The saccharin preference of the sham-operated controls decreased as compared to the Vest-Dam group on the second and third test sessions (ps $<.05$ ), but this difference was not maintained on the following two preference tests (ps $>.05)$. These results are in accord with findings reported by Braveman (1975) and Cannon et al. (1975), who also found that prior experience with the UCS in the absence of associated taste experience

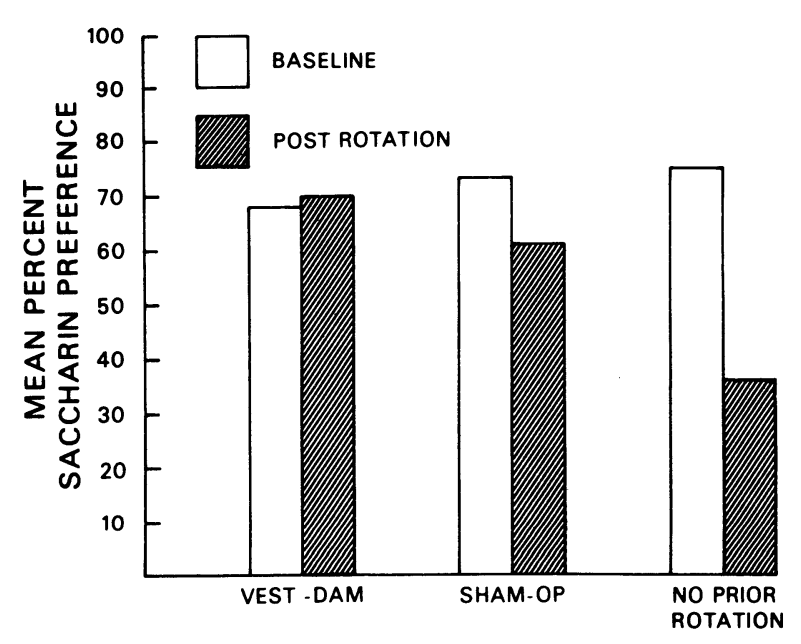

Figure 3. Mean percent saccharin preference for all three groups on prerotation baseline and subsequent postrotation preference sessions. 
attenuated the efficacy of that UCS in producing taste aversions.

\section{EXPERIMENT 3b}

In the preceding experiment, both experiential manipulations (prior exposure to the UCS) and physiological insult (vestibular destruction) substantially alleviated rotation-induced taste aversion. One concern which arises, however, is whether the failure to obtain taste aversion specifically reflects the changes in responsiveness to vestibular stimulation, rather than a more general associative deficit or possibly a methodological artifact. Demonstrating that the same subjects are indeed capable of acquiring taste aversion under other conditions would effectively eliminate these alternatives. Toward this end, two separate manipulations were carried out. Initially, we attempted to establish taste aversion in the intact animals (Sham-Op) simply by reversing the direction of rotation from clockwise to counterclockwise on the assumption that habituation produced by prior treatments might be relatively specific to the directional component of treatment. If intact rats had increased tolerance to a specific rotation condition, then exposing them to the opposite angular velocities should yield malaise and learned taste aversion. For the operated animals, rotational direction should be irrelevant, of course, and, as in the preceding experiment, no taste aversion would be expected. When it became apparent that taste aversion based on motion sickness was not acquired by either group, the subjects were given $\mathrm{LiCl}$ poisoning as the UCS in lieu of rotation.

\section{Method}

Subjects. The vestibular damage and sham-operated groups were used again for this experiment. The housing, food, and water-ration conditions of the previous experiment were maintained.

Apparatus. The apparatus used in this experiment consisted of the rotation turntable previously described, but rotation was counterclockwise. A $.15 \mathrm{M}$ solution of $\mathrm{LiCl}$ was used as the poisoning agent.

Procedure. Following the fifth saccharin-rotation test cycle of Experiment 3a, the 4-day cycle of taste-UCS-test was repeated once more; however, the subjects were rotated in the opposite (counterclockwise) direction. Two more 4-day cycles followed, but on these occasions, in place of rotation, $2 \mathrm{ml} / 100 \mathrm{~g}$ body weight of $.15 \mathrm{M} \mathrm{LiCl}$ was administered IP immediately following saccharin consumption.

\section{Results and Discussion}

Reverse (counterclockwise) rotation was ineffective in producing any decrease in the saccharin preference of the Vest-Dam group. A comparison of saccharin preference before and after reverse rotation revealed no statistically significant differences $(\mathrm{t}=1.39$, $\mathrm{p}>.05)$. Reverse rotation was also ineffective in producing taste aversion in the intact, Sham-Op, group $(t=1.07, p>.05)$, suggesting an associational

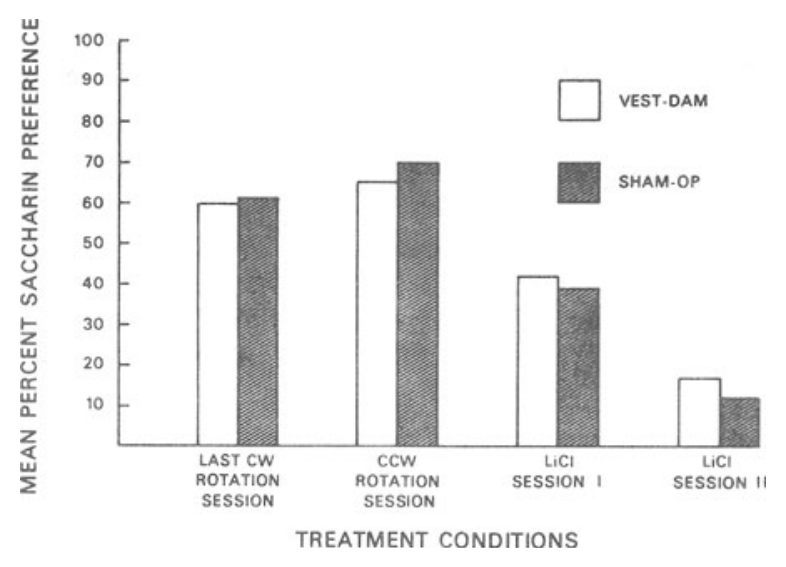

Figure 4. Mean percent saccharin preference following clockwise and counterclockwise rotation and after two lithium chloride poisoning sessions for both Vest-Dam and Sham-Op groups.

failure based on prior motion sickness experience (cf. Braveman, 1975).

As Figure 4 indicates, when the UCS was changed from rotation to $\mathrm{LiCl}$ poisoning, however, substantial decreases in the saccharin preference of both groups occurred. A comparison of pre- and postpoisoning saccharin preferences revealed a significant and equal decrease in the saccharin preferences of both Sham-Op and Vest-Dam groups (t tests, ps <.05). The dramatic decrease in the saccharin preference of the Vest-Dam group following poisoning indicates that the lack of rotation-induced taste aversion in the earlier experiment was not attributable to sensory/associative impairment or insensitive methodology, as the aversion was learned rapidly in both groups when $\mathrm{LiCl}$ was the UCS.

\section{GENERAL DISCUSSION}

The first two experiments demonstrate that a posttreatment recovery measure can provide a useful index of the severity of rotation-induced sickness. Even with a relatively restricted range of session lengths ( 2 to $10 \mathrm{~min}$ ), the suppression of postrotation water intake was found to vary directly as a function of the duration of treatment. This finding is consistent with evidence, recently reported by Green and Rachlin (1976), that the magnitude of conditioned taste aversion also increases with longer rotation sessions. Moreover, when treatment length was held constant, the disruption of drinking measure proved sensitive to the type of rotation employed. Repeated accelerations and decelerations apparently produce a greater degree of motion sickness than continuous exposure to a constant angular velocity. That vestibular mechanisms are involved in producing the putative UCR of motion sickness was substantiated by data showing that operated rats did not 
exhibit the typical elevation of drinking latencies following rotation.

Since independent evaluation of the UCR is a relatively neglected aspect of many taste aversion studies, the present technique (or variants thereof) may prove generally useful. For example, by allowing an assessment of malaise independent of the taste aversion outcome, the recovery index may permit more systematic comparisons of various illnessinducing agents. Evidence that taste aversion can occur following treatments which ostensibly do not involve illness (e.g., Berger, 1972; Smith, 1971), in conjunction with the failure to establish aversions with apparently highly toxic agents (Nachman \& Hartley, 1975), suggests that such independent assessment may be warranted. Moreover, determining the duration of the UCR may provide information relevant to certain theoretical interpretations of the taste aversion effects. Recently, Krane and Wagner (1975) proposed that differences in the ease of establishing Pavlovian conditioning with long CS-UCS intervals when exteroceptive or interoceptive events are employed may reflect quantitative rather than qualitative characteristics. In brief, they suggest that trace characteristics of the CS may interact with duration of the UCS (and presumably UCR) to influence conditioning. Thus, persistence of a gustatory (trace) CS after termination of an exteroceptive UCS (shock) is considered to impair excitatory conditioning. In this connection, then, the present finding that rotationinduced malaise is relatively brief becomes of particular interest. While suppression of drinking lasted for only several minutes following $10 \mathrm{~min}$ of intermittent rotation, this same treatment is effective in producing learned aversion to saccharin with at least a 30-min CS-UCS delay (Haroutunian \& Riccio, 1975). We recognize that the choice of baseline conditions such as degree of deprivation may markedly affect the measured "duration" of sickness in this situation, but there seems little doubt that rotation effects are substantially less prolonged than those produced by more typical poisoning agents. Indeed, it remains an empirical question as to whether the disruptive effects of footshock subside much faster than do those of rotation.

The present study also provides direct evidence for the role of the vestibular system in rotation-induced taste aversion. In the absence of histological verification, the extent of labyrinthine damage cannot be determined. Destruction was sufficient, however, as the operated rats failed to show postrotation suppression of drinking (Experiment 2) and did not acquire an aversion when a novel flavor was paired with rotation. As noted above, the lack of taste aversion did not represent a general associative failure, since these rats did learn an aversion when toxicosis served as the UCR. Consistent with several other recent studies (Braveman, 1975; Cannon et al., 1975), we also found that prior noncontingent rotation attenuated the establishment of conditioned taste aversion. It is of interest that while this proactive effect was independent of the direction of rotation at treatment the protection did not transfer to a taste aversion paradigm using a different type of illness-inducing agent. This outcome suggests that there may well be important qualitative differences in "malaise" that depend upon the eliciting stimulus. A similar suggestion has recently been made by Nachman and Hartley (1975), who demonstrated that several poisons which produced clear signs of sickness and debilitation nevertheless failed to support the establishment of taste aversion.

\section{REFERENCES}

Berger, B. D. Conditioning of food aversions by injections of psychoactive drugs. Journal of Comparative and Physiological Psychology, 1972, 81, 21-26.

BraUn, J. J., \& McINTOSH, H. Learning taste aversions induced by rotational stimulation. Physiological Psychology, 1973, 1, 301-304.

Braveman, N. S. Formation of taste aversion in rats following prior exposure to sickness. Learning and Motivation, 1975, 6, 512-534.

Cannon, D. S., Berman, R. F., Baker, T. B., \& Atrinson, C. A. Effect of preconditioning unconditioned stimulus experience on learned taste aversions. Journal of Experimental Psychology: Animal Behavior Processes, 1975, 104, 270-284.

Eskin, A., \& Riccio, D. C. The effects of vestibular stimulation on spontaneous activity in the rat. Psychological Record, 1966, 16, 523-527.

GrEen, L., \& RACHLIN, H. The effect of rotation on the learning of taste aversions. Bulletin of the Psychonomic Society, 1973, 1, 137-138.

GreEN, L., \& RACHLIN, H. Learned taste aversions in rats as a function of delay, speed, and duration of rotation. Learning and Motivation, 1976, 7, 283-289.

Haroutunian, V., \& Riccio, D. C. Acquisition of rotationinduced taste aversion as a function of drinking-treatment delay. Physiological Psychology, 1975, 3, 273-277.

Johnson, W. H., MeEk, J. C., \& Graybiel, A. The effects of unilateral and bilateral labyrinthectomy on canal sickness in the squirrel monkey. Annals of Otology, Rhinology, \& Laryngology, $1962,71,289-298$.

KRANE, R., \& WAgNeR, A. R. Taste aversion learning with a delayed shock UCS: Implications for the "generality of the laws of learning." Journal of Comparative and Physiological Psychology, 1975, 88, 882-889.

Nachman, M., \& HaRTley, P. L. Role of illness in producing learned taste aversions in rats: A comparison of several rodenticides. Journal of Comparative and Physiological Psychology, 1975, 89, 1010-1018.

Riccio, D. C., IgARASHI, M., \& Eskin, A. Modification of vestibular sensitivity in the rat. Annals of Otology, Rhinology, Laryngology, 1967, 76, 179-189.

Riccio, D. C., \& TнACH, J. S. Rotation as an aversive stimulus for rats. Psychonomic Science, 1968, 5, 267-268.

SMITH, J. C. Radiation: Its detection and its effects on taste preferences. In E. Stellar \& J. M. Sprague (Eds.), Progress in physiological psychology (Vol. 4). New York: Academic Press, 1971.

W ANG, S. C., \& CHINN, H. I. Experimental motion sickness in dogs: Importance of labyrinth and vestibular cerebellum. American Journal of Physiology, 1956, 187, 617-623.

(Received for publication July 20, 1976; revision accepted August 19, 1976.) 